\title{
First case of Echinococcus vogeli infection imported to the Netherlands, January 2013
}

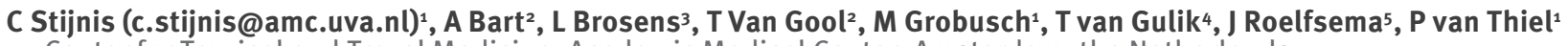

1. Center for Tropical and Travel Medicine, Academic Medical Center, Amsterdam, the Netherlands

2. Department of Medical Microbiology, Section Parasitology, Academic Medical Center, Amsterdam, the Netherlands

3. Department of Pathology, Academic Medical Center, Amsterdam, the Netherlands

4. Department of Surgery, Academic Medical Center, Amsterdam, the Netherlands

5. National Institute for Public Health and the Environment (RIVM), Bilthoven, the Netherlands

Citation style for this article:

Stijnis C, Bart A, Brosens L, Van Gool T, Grobusch M, van Gulik T, Roelfsema J, van Thiel P. First case of Echinococcus vogeli infection imported to the Netherlands, January 2013. Euro Surveill. 2013;18(15):pii=20448. Available online: http://www.eurosurveillance.org/ViewArticle.aspx?Articleld=20448

Article submitted on 28 March 2013 / published on 11 April 2013

In January 2013 in the Netherlands, a man in his 505 from Suriname underwent hemihepatectomy because of a cystic liver mass, assumed to be a cystadenoma. Pathology revealed an echinococcal infection. PCR analysis of cyst material identified Echinococcus vogeli, causing polycystic hydatid disease. This echinococcus species is rarely diagnosed outside South America. The patient received adequate treatment, but this case emphasises the importance of awareness of this infection when treating patients with cystic tumours from endemic areas.

\section{FIGURE 1}

Computed tomography scan of the abdomen of a patient with Echinococcus vogeli infection, the Netherlands,

January 2013

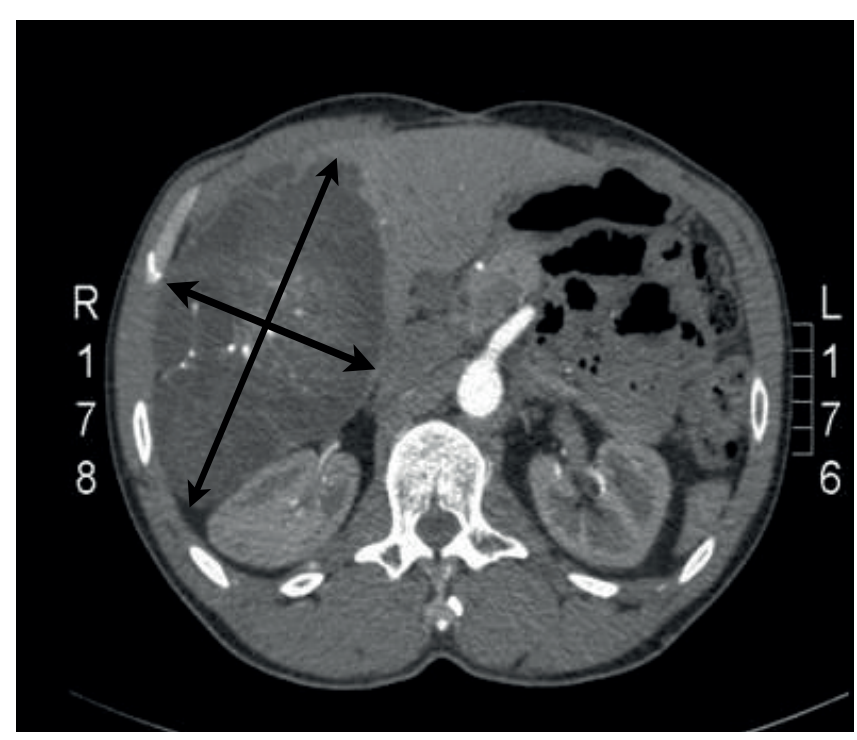

The arrowed lines indicate the extent of the cyst.

\section{Case description}

In October 2012, a man in his 50 of of Surinamese descent noticed a painless mass in his right upper abdomen and was referred to the surgical department of the Academic Medical Center in Amsterdam, the Netherlands. Until 1984, he had been living in Suriname, where his work in hydrography entailed exploring all rivers in this country, frequently under primitive conditions. His medical history was otherwise unremarkable. Physical examination confirmed a palpable mass in the right abdomen. Routine haematology laboratory testing was normal, except for a slightly elevated percentage of eosinophils in the white blood cell differential (o.65\%, no absolute count was performed). Liver biochemistry tests and alpha1-fetoprotein were not elevated. Imaging (abdominal computed tomography scan, Figure 1) showed a solitary, large multilocular cyst in the right side of the liver with diffuse calcifications.

\section{FIGURE 2}

Perioperative photograph of right hemihepatectomy of a patient with Echinococcus vogeli infection, the Netherlands, January 2013

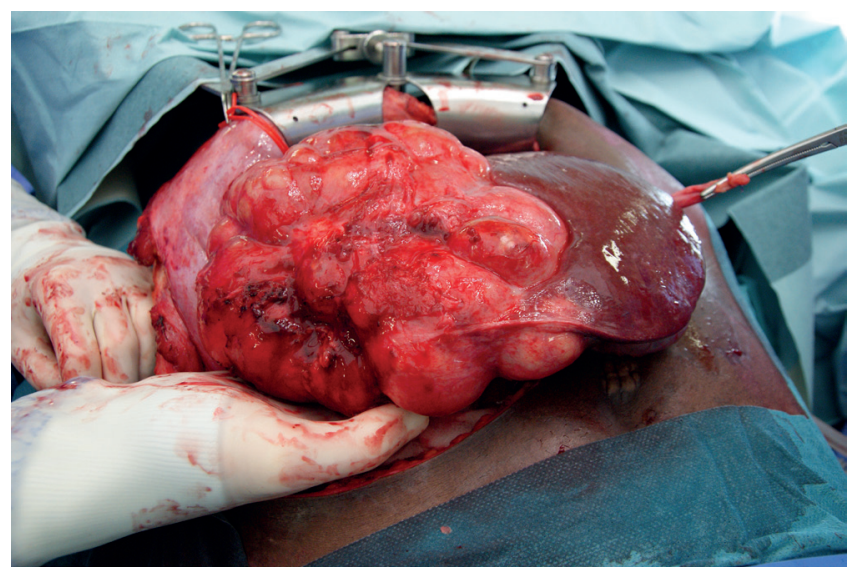




\section{FIGURE 3}

Histopathological examination of the liver of right hemihepatectomy of a patient with Echinococcus vogeli infection, the Netherlands, January 2013
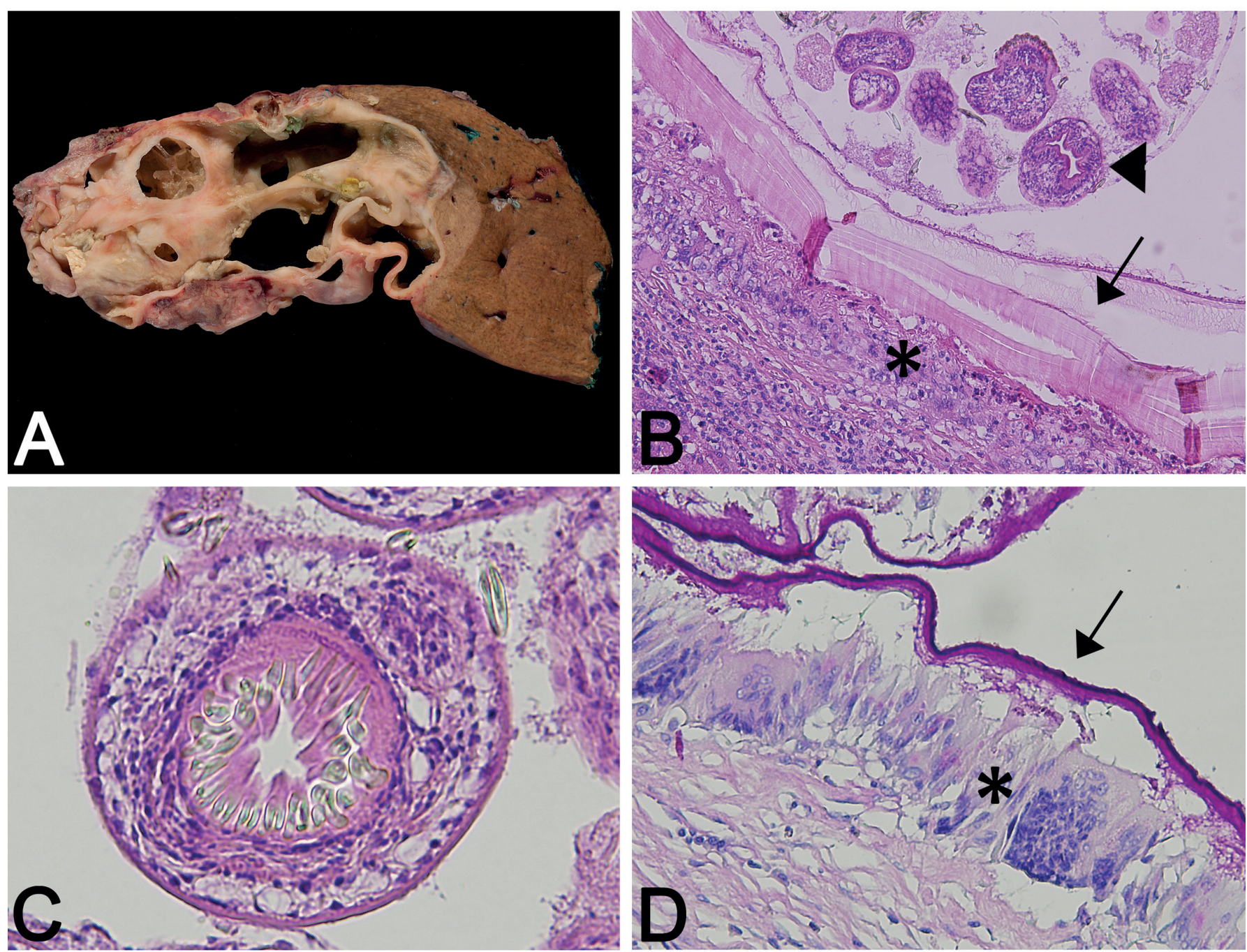

A. Section of the liver showing a multilocular cystic tumour (diameter $18 \mathrm{~cm}$ ).

B. Microscopic examination (hematoxylin and eosin stain, 100x) showing cystic spaces lined by laminated membranes (arrow) and surrounded by a rim of histiocytes (asterix). The cystic spaces were filled with necrotic debris and numerous protoscolices (arrowhead).

C. Detail of protoscolix with hooklets (hematoxylin and eosin stain, 400x).

D. Periodic acid-Schiff (PAS) stain (200x) showing laminated PAS-positive membranes (arrow) and rim of histiocytes and multinucleated giant cells facing the hydatid cysts (asterix). 
Because a cystadenoma with possible malignant degeneration was suspected, he underwent right hemihepatectomy (Figure 2), in January 2013, which was successful. Much to the surprise of the surgeon, microscopic examination of the cyst fluid revealed protoscolices and hooklets, consistent with echinoccocal infection.

Histopathological examination of the liver showed a multilocular cystic tumour with a diameter of $12 \mathrm{~cm}$. The cystic spaces were filled with necrotic debris, numerous protoscolices and hooklets and laminated periodic acid-Schiff-positive membranes. The lesion was surrounded by fibrosis with eosinophilic and lymphoplasmacytic inflammatory infiltrate and a rim of histiocytes and multinucleated giant cells directly facing the hydatid cysts (Figure 3).

Post-operative Echinococcus serology [1] was positive (indirect haemagglutin test 1 : 320). Because of the atypical nature of the cyst, a specific polymerase chain reaction (PCR) was performed on cyst material., amplifying the mitochondrial $12 \mathrm{~S}$ rRNA gene. The primers used were derived from the PCR developed by Trachsel et al. [2]. The reverse primer, Cest12Sr (GCGGTGTGTACITGAGITAAAC) was basically the same as Cest5 from Trachsel et al., but the forward primer, Ech12Sf (AAAIGGTTTGGCAGTGAGIGA) was designed by ourselves (unpublished data). The PCR results in a product of 285 nucleotides. Sequence analysis of the PCR product showed important similarity to Echinococcus vogeli (Figure 4).

\section{Background}

Most patients with echinococcis in the Netherlands are migrants from eastern Europe, northern Africa, Turkey and the Middle East and are diagnosed with cystic echinococcosis. The so-called hydatid cysts are caused by Echinococcus granulosus sensu lato, a tapeworm of dogs, the definite hosts. Livestock (cattle, pigs, goats, sheep, camels) become intermediate hosts* after ingestion of eggs, with cyst formation in visceral organs. Infected humans can become intermediate (but dead-end) hosts themselves, with cysts in several organs as well. The liver is most affected, but sometimes also the spleen, lungs, muscles, bones and even the central nervous system are involved. The cysts may cause pain or discomfort by their size, but are often asymptomatic and coincidentally diagnosed. Cyst rupture into the abdominal cavity is an uncommon but life-threatening complication, resulting in anaphylaxis and seeding of the infection. This may also be caused by surgical perforation of cysts [3].

\section{Discussion}

In the Center for Tropical and Travel Medicine in the Academic Medical Center in Amsterdam, a total of 133 patients with echinococcal disease have been treated in the last decade, with approximately 20 new cases being referred each year [4]. Although other hospitals in the Netherlands also diagnose new cases, most patients are referred to the Academic Medical Center because of its specific expertise in ultrasound diagnosis and treatment with percutaneous techniques and adjuvant chemotherapy with albendazol [5].

\section{FIGURE 4}

Sequence comparison of the mitochondrial 12S rRNA gene sequences of Echinococcus species from GenBank and from a patient with E. vogeli infection**, the Netherlands, January 2013

\begin{tabular}{|c|c|c|c|c|c|c|c|}
\hline & تホสำ & 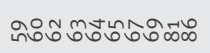 & & 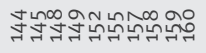 & 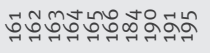 & ㄱำกีก & Accession number \\
\hline Patient & tttagaaaat & g-gtgatatg & ttga-gaatg & tggtaggaac & at--ga-gag & gtgactc & KC894948 \\
\hline E. vogeli & $\ldots \ldots \ldots$ & $-\ldots \ldots$ & $\ldots \ldots$ & $\ldots \ldots$ & $\ldots--\ldots-\ldots$ & $\ldots . .9$ & DQ408426.1 \\
\hline E. equinus & $.9 \ldots \ldots$ & - -a.a...ga & ...-a..g. & ..t.tt...t & $\ldots--t .-t \ldots$ & . a....g & EF143835.1 \\
\hline E. gran_buffel & $\ldots g \cdot \ldots g \cdot c$ & $t-a \cdot . g \cdot g \cdot$ & c.ag-a..g. & .a.tttg.t & $\ldots--t .-t \cdot a$ & a.ag..g & GQ168813.1 \\
\hline E. ortleppi & $\ldots g \cdot \ldots g \cdot c$ & $t-a \cdot . g \cdot g \cdot$. & c.ag-a..g. & ..a.tttg.t & ..--t. -t.a & a.ag...g & FJ608743.1 \\
\hline E. can-G6 & . c...g. c & $t-a \cdot \cdot g \cdot g \cdot$ & c..g-a..g. & ....tttg.t & $\cdot--t \cdot-t \cdot t$ & $a \ldots . g$ & $A B 235846.1$ \\
\hline E. can-G7 & .....g.c & $t-a \cdot \cdot g \cdot g \cdot \cdot$ & c..g-a..g. & ....tttg.t & $\cdots--t \cdot-t \cdot t$ & $a \ldots . g$ & EU541210.1 \\
\hline E. can-G8 & . c . . . c c & t-a..g.g.. & c..g-a..g. & ....tttg.t & $\ldots--t \cdot-t \cdot t$ & $\mathrm{a} \cdot \mathrm{a} . \mathrm{cg}$ & $A B 235847.1$ \\
\hline E. multi & .a..t.g... & $t-\ldots a \cdot g \ldots$ & ..a.-. gaa & .a..tttggt & $\ldots--\operatorname{tg}-\operatorname{tg} t$ & $t \ldots . g$ & $A B 235848.1$ \\
\hline E. gran_sheep & $.9 . . t .9 g \ldots$ & $-a \cdot a \ldots$. & . tg-a..g. & .aa.t.tg.t & $g \cdot--. .-t \ldots$ & . a... g & EU043371.1 \\
\hline E. oligarthrus & $a \ldots .$. &.- a..... & $. g . .-. t . . a$ & a..a...tgg & $\operatorname{tg}-t \cdot t-t$. & ta.... & AJ237779.1 \\
\hline E. felidis & $.9 . . \operatorname{ag} \ldots$ & .sa...... & ...gsa..g. & ...t.tggt & $\ldots--. . t \cdot t$ & ta..g.g & FJ426641.1 \\
\hline
\end{tabular}

Alignment of polymorphic sites in the alignment of 230 nucleotides of the $12 \mathrm{~S}$ rRNA gene sequence of various Echinococcus species retrieved from the GenBank nucleotide sequence database (accession numbers DQ408426.1, EF143835.1, GQ168813.1, FJ608743.1, AB235846.1, EU541210.1, AB235847.1, AB235848.1, EU043371.1, AJ237779.1, and FJ426641.1). The position of the polymorphic sites is indicated relative to position 94 in E. vogeli $12 \mathrm{~S}$ rRNA gene with GenBank accession number DQ408426.1. The number of polymorphisms clearly shows similarity of the patient's sequence to the $E$. vogeli sequence in GenBank, and dissimilarity to other Echinococcus species. Identical positions are indicated with a dot, alignment gaps are indicated with a dash.

**The GenBank accession number was added on 17 April 2013. 
Alveolar echinococcosis is caused by Echinococcus multilocularis, a tapeworm favouring the fox as a definite host. This is a more aggressive type of infection, often necessitating surgery and lifelong chemotherapy. Recently, a first case of probable locally acquired E. multilocularis in the Netherlands has been described [6]; in Denmark, the parasite has been detected in fox populations [7].

E. vogeli, as described in our patient, occurs in South America [8-13], including Suriname [8,13], as a tapeworm of the bush dog, with rodents as intermediate hosts. In humans, it may cause polycystic hydatid disease and is described in a small, but growing number of patients from this area [8-13]. To the best of our knowledge, this is the first report of $E$. vogeli in the Netherlands and probably also in Europe. As shown in our patient, the diagnosis is easily missed and may be mistaken for malignancy. Fortunately, the correct therapy was administered: radical surgery with no spill of cystic content, to be followed by at least six months of albendazol and lifelong surveillance. However, if this diagnosis had been considered before the operation, therapy with albendazol would probably have been started pre-operatively and antihistamnics administered peri-operatively.

\section{Conclusion}

In patients originating from endemic areas who have otherwise unexplained cystic masses, especially in the liver, the differential diagnosis must include echinococcosis. Most such patients in the Netherlands are diagnosed with $E$. granulosus. The case described here indicates that other pathogenic species such as E. vogeli, causing a different type of cystic disease, should also be considered. This, and earlier reports [8-13] from South America, show that E. vogeli is probably endemic to some parts of this continent.

\section{Acknowledgements}

Joke van der Giessen and Titia Kortbeek (National Institute for Public Health and the Environment (RIVM), Bilthoven, The Netherlands) for their advice, based on extensive expertise of echinococcosis.

\section{Conflict of interest}

None declared.

\section{Authors' contributions}

Cornelis Stijnis wrote the article. Aldert Bart, Lodewijk Brosens, Martin Grobusch, Thomas van Gulik, Jeroen Roelfsema and Pieter van Thiel revised drafts of the manuscript. Lodewijk Brosens analysed pathology results and produced the pathology figure. Tom van Gool analysed serology results. Thomas van Gulik performed surgery and postoperative photography. Jeroen Roelfsema analysed PCR results and produced the PCR figure.
* Authors' correction:

The authors are grateful to a reader for spotting an error in the background information. Rodents are intermediate hosts for Echinococcus multilocularis, not for Echinococcus granulosus. This was corrected on 16 April 2013.

** Addendum

The GenBank accession number was added to Figure 4 on 17 April 2013.

\section{References}

1. van Doorn HR, Hofwegen H, Koelewijn R, Gilis H, WentinkBonnema E, Pinelli E, et al. Reliable serodiagnosis of imported cystic echinococcosis with a commercial indirect hemagglutination assay. Diagn Microbiol Infect Dis. 2007;57(4):409-12.

http://dx.doi.org/10.1016/j.diagmicrobio.2006.10.002. PMid:17189677.

2. Trachsel D, Deplazes $P$, Mathis A. Identification of taeniid eggs in the faeces from carnivores based on multiplex PCR using targets in mitochondrial DNA. Parasitology. 2007, 134(Pt 6):911-20.

3. Kern P. Echinococcus granulosus infection: clinical presentation, medical treatment and outcome. Langenbecks Arch Surg. 2003;388(6):413-20. http://dx.doi.org/10.1007/ s00423-003-0418-y. PMid:14605887.

4. Herremans T, Verweij JJ, Schipper HG, Casparie M, van Lieshout L, Pinelli E, et al. [Decline of echinococcosis in the Netherlands; 1997-2008]. Ned Tijdschr Geneeskd. 2010. 154(18):A2297. Dutch.

5. Schipper HG, Laméris IS, van Delden OM, Rauws EA, Kager PA. Percutaneous evacuation (PEVAC) of multivesicular echinococcal cysts with or without cystobiliary fistulas which contain non-drainable material: first results of a modified PAIR method. Gut. 2002;50(5):718-23. http://dx.doi.org/10.1136/ gut.50.5.718. PMid:11950823. PMCid:1773202.

6. van Dommelen L, Stoot JH, Cappendijk VC, Abdul Hamid MA, Stelma FF, Kortbeek LM, et al. The first locally acquired human infection of Echinococcus multilocularis in the Netherlands. J Clin Microbiol. 2012;50(5):1818-20. http://dx.doi.org/10.1128/ JCM.06355-11. PMid:22357506. PMCid:3347149.

7. van der Giessen JW, Rombout YB, Franchimont JH, Limper LP, Homan WL. Detection of Echinococcus multilocularis in foxes in the Netherlands. Vet Parasitol. 1999;82(1):49-57. http://dx.doi. org/10.1016/S0304-4017(98)00263-5

8. Basset D, Girou C, Nozais IP, D'Hermies F, Hoang C, Gordon R, et al. Neotropical echinococcosis in Suriname: Echinococcus oligarthrus in the orbit and Echinococcus vogeli in the abdomen. Am J Trop Med Hyg. 1998;59(5):787-90.

9. D’Alessandro A, Rausch RL. New aspects of neotropical polycystic (Echinococcus vogeli) and unicystic (Echinococcus oligarthrus) echinococcosis. Clin Microbiol Rev. 2008;21(2):380-401. http://dx.doi.org/10.1128/CMR.00050-07. PMid:18400802. PMCid:2292577.

10. Knapp J, Chirica M, Simonnet C, Grenouillet F, Bart JM, Sako $Y$, et al. Echinococcus vogeli infection in a hunter, French Guiana. Emerg Infect Dis. 2009;15(12):2029-31. http://dx.doi. org/10.3201/eid1512.090940. PMid:19961693. PMCid:3044547.

11. Somocurcio JR, Sánchez EL, Náquira C, Schilder J, Rojas F, Chacón $\mathrm{P}$, et al. First report of a human case of polycystic echinococcosis due to Echinococcus vogeli from neotropical area of Peru, South America. Rev Inst Med Trop Sao Paulo. 2004;46(1):41-2. http://dx.doi.org/10.1590/Soo3646652004000100008. PMid:15057334.

12. Tappe D, Stich A, Frosch M. Emergence of polycystic neotropical echinococcosis. Emerg Infect Dis. 2008;14(2):292 7. http://dx.doi.org/10.3201/eid1402.070742. PMid:18258123 PMCid:2600197.

13. Oostburg BF, Vrede MA, Bergen AE. The occurrence of polycystic echinococcosis in Suriname. Ann Trop Med Parasitol. 2000;94(3):247-52. http://dx.doi. org/10.1080/00034980050006429. PMid:10884869. 